\title{
Elementary excitations of Ising models at the critical temperatures
}

\author{
You-Gang Feng \\ College of Science, Guizhou University, Huaxi, Guiyang, 550025 China
}

\section{Email address:}

ygfeng45@aliyun.com

\section{To cite this article:}

You-Gang Feng. Elementary Excitations of Ising Models at the Critical Temperatures. American Journal of Modern Physics. Vol. 3, No. 6, 2014, pp. 211-217. doi: 10.11648/j.ajmp.20140306.11

\begin{abstract}
Ordered Ising models as ferromagnetic having nonsingular heat capacities at the critical temperatures are considered. A new parameter vector $\vec{q}$ is found to describe the spin correlations and fluctuation characteristics. The conservation of scalar $q$ indicates that there is simple harmonic motion of $\vec{q}$, and the motion's quantum is called block-spin phonons, like the phonons in a crystal, resulting in nonsingular heat capacity near the critical point. The harmonic motion shows there are hierarchies and symmetries of fluctuations, while the soft mode may lead to the interactions of block-spin phonons with different frequencies. We are certain that the critical point for an Ising model only exists in a statistical sense although the system at the critical temperature. The fluctuations undergo about the critical point, which the system never attains. It is the first time for us that the specific forms of the spins' correlation functions for Ising models at the critical temperatures are obtained.
\end{abstract}

Keywords: Ising, Correlation, Phonon, Heat Capacity, Fluctuation

\section{Introduction}

The critical phenomena are characterized by the free energy singularity and the long-range correlations of spins. The former indicates that an old phase, a disordered state, disappears; the latter shows that this is just the property of a new phase, an ordered state. As a new state it is considered a normal ferromagnetic that should have its normal and nonsingular free energy and heat capacity. It is incredible that a ferromagnetic Ising model has no normal thermodynamic quantities to which the spins contribute. There has been only one theory so far, the spin-wave theory, can explain these normal quantities. Freeman J. Dyson drew a physical picture for a 3-di-mensional model [1]. On microscopic level, as his showing, the spin waves arise from interference effects in the lowest partial-wave collisions resulting in a rotation of the spins of the scattered atoms. The cumulative effect of many such rotating collisions is such that inhomogeneous spin states propagate like waves rather than diffusively. Dyson presented that his theory was suitable to the low temperature. V. G. Vaks and his colleagues tried to apply the theory to a Heisenberg model to study the spin waves and correlation function [2]. They found that the damping of the spin waves and the damping increased with the increasing temperature in the range $T<T_{c}$. All of these tell us that the spin-wave picture cannot illustrate the critical phenomena, especially the critical properties of an Ising model, since there is no spin-spin collision caused by the moving atoms with spins. The authors of the reference [3] constructed an inhomogeneous planar square lattice Ising model with finite size, and discussed its abnormal specific heat at the critical temperature under a boundary condition. We think that, however, there is great deference between such model and conventional Ising model. The rest of the model is still infinite if a finite part is picked up from an infinite Ising model. In addition, we notice that the authors' work is prior to the Wilson's renormalization group theory, and the imposed boundary condition makes the work be considered as a particular result rather than general. Some coherent spin states similar to linear harmonic oscillators were introduced by J. M. Radcliffe [4], and the relevant physical properties were discussed for a Heisenberg model. He supposed that such discussion might benefit to the understanding of the correlation of spins. His idea may inspire us to investigate the underlying form of the spin's correlation function.

In this paper we try to show by our theory the normal properties of the new phase for Ising models at $T_{c}$. In section 
2 we find a spin parameter vector $\vec{q}$ describing the block spin correlations and get a conservation equation of the scalar $q$, revealing the block-spin correlations exist in the form of simple harmonic waves. As a result we get the quantum of the wave motion, the block-spin phonon. In section 3 we obtain nonsingular heat capacities for the new phase and discuss the correlation functions with some symmetric properties and interpret the hierarchies in the fluctuations. We also consider the occurrence of soft modes. Section 4 is conclusion.

This article is a revision of the reference [5], it is not only a continuation of the reference [6] but also a base for the reference [7].

\section{Theory}

\subsection{Spin Parameter Vector $\vec{q}$}

The calculating results of the critical points for the 2dimansional models and 3-dimensional models show that our theory is available for the investigation of the critical behaviors of Ising models [6]. Especially, the fractal analysis used in this theory reveals the detailed structures in the fluctuations, which has never been discussed in other theories. The concepts of the block spin and the self-similar transformation mentioned in this article all are attributed to this theory. According to this theory, a system will never arrive at its critical point $K_{c}=j /\left(k_{B} T_{c}\right)$ due to that the selfsimilar transformation forbids the fractional side $n^{*}$. The Eq. (11) of the reference [6] indicates that the free energy of the system dependents on the magnitude of the logarithm on the right side of the equation, the smaller the value of the logarithm the lower the free energy because the natural logarithm is a monotone function. In order to minimize the free energy the system always try to attain the critical point such that the two terms in the logarithm should be close to each other, which leads to that the system adjust the block's side from time to time. Such adjustment will never stop, which is just the cause of the endless fluctuation. Let's consider two limit cases. At first, for a finite number $r$, on the $\mathrm{r}$-th hierarchy the vector summation of block spins is always zero although the spins are correlative with each other. In the meantime a system appears order only on the infinite hierarchy, namely, $r \rightarrow+\infty$, where the system is just an isolated block spin after infinite iteration of the transformations. Denote the system magnetization by $\vec{M}_{1}$. In the second case, on a finite hierarchy all block spins are parallel to each other, consequently the system is order. Denote the relevant magnetization by $\vec{M}_{2}$. Clearly, the magnitude of $M_{1}$ is smaller than the magnitude of $M_{2}$. So the $\vec{M}_{1}$ is connected with $T_{c}$, and the $\vec{M}_{2}$ with the temperature $\mathrm{T}$ lower than $T_{c}$. The fluctuations are not only the deviation in the block sides, but also the deviation in the block-spin states. An obvious disadvantage of the conventional spin parameter $\vec{S}$ is that it is always one- dimension such that it cannot be a match for the spin correlations that have the same dimensions as the ones of the lattice system. In order to research for the fluctuation nature further we should find a new spin parameter of d-dimensions, besides $\vec{S}$, to describe the correlations. Solving the Gaussian model in the reference [6] we introduce a parameter vector $\vec{q}$ in the Fourier transform, a new lattice spin is $S_{i}=(1 / \Omega) \sum_{q} S_{q} \exp \vec{q} \cdot \vec{r}_{i}$. The vector $\vec{q}$ is originally a reciprocal lattice vector for the new lattice but the one for a block spin system. The so-called new lattice spin is actually the lattice spin on the $i$-th hierarchy, which is just the block spin on the $(i-1)$-th hierarchy. The magnitude of $\vec{q}$ determines the new lattice spin magnitude being consistent with the magnitude of a certain block spin, its changes in both direction and magnitude relate to the changes of the new lattice spins in both direction and magnitude. Because there is a mapping relationship between a new lattice spin and a block spin, if the vector $\vec{q}$ can serve as an appropriate parameter to demonstrate the block spin state for a certain block-spin system instead of the new lattice spin system, it should have specific features that there is an one-to-one correspondence between the value of $\vec{q}$ and the magnitude $\mathrm{S}$ of a block spin, each block spin has its own $\vec{q}$, and all of block spins with $\vec{q}$ are correlated. The vector change in direction is connected with the block spin change in direction. The traversal time of the component of $\vec{q}, q_{x}$, or $q_{y}$, or $q_{z}$, in either its own positive-direction state or negative-direction state is identical, since every block spin has the same probability in both the spin-up state and the spin-down state in the thermodynamic equilibrium on any finite hierarchy to keep the spin vector summation zero. In a word, the $\vec{q}$ will be a d-dimensional periodically varying parameter rather than random.

\subsection{Conservation Equation of Scalar $\vec{q}$}

On the one hand the free energy will be singularity at the critical point $K_{c}=j /\left(k_{B} T_{c}\right)$; on the other hand the fluctuations undergo around the critical point, such that the magnitude of the block spin is not the minimum related to the critical point, and q doesn't vanish at $T_{c}$, since only the $q=0$ links to $K_{c}$. Therefore, the algebraic expression the equation (10) of [6] changes into $K(\vec{q})=1 / S_{t r}{ }^{2}$, and $S_{t r}{ }^{2}>S_{t r, \min }^{2}$, for the triangle lattice spin system, where $K(\vec{q})=K \sum_{\delta_{i j}} \exp -i \vec{q} \cdot \vec{\delta}_{i j}, \vec{\delta}_{i j}$ is a vector from the $i$-th lattice to the $j$-th lattice, and $K=j /\left(k_{B} T\right)$. Generally, for a 2dimensional system when the integer side $n$ varies about the fractional side $n^{*}$ the value of $q$ changes certainly around $q=0$. Since the magnitude of $q$ is very small we can 
expand the function $K(\vec{q})$ about $q=0$ as a power series to keep its quadratic term of $q$. We then get (see the appendix)

$$
q_{x}^{2}+q_{y}^{2}=\frac{4}{(n+1)^{2}}\left[1-\frac{D_{\min }}{D}\right]
$$

where the fractal dimension $D_{\text {min }}$ is determined by the fractional side $n^{*}$, the fractal dimension $D$ is determined by the integer side $n$. Equation (1) signifies that there is a circle of radius $\mathrm{q}$ and a rotating vector $\vec{q}$ (see Figure 1), and the initial point and the terminal point of the vector are at the circle center and on the circumference, respectively. When the vector rotates its direction changes, the rotation is a unique

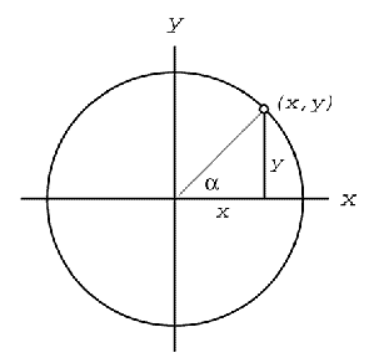

Figure 1. A circle of radius $q$, where the symbol $x$ refers to $q_{x}$, and y to $q_{y}$.

motion way for it. Clearly, if the side is constant the magnitude of $q$ is certain too; and vice versa. The conservation of scalar $q$ corresponds to the constant block spin value. The following trigonometric functions are the solutions of Eq. (1):

$$
\begin{aligned}
& q_{x}=q \cos [\omega(t \mp r / v)+\alpha] \\
& q_{y}=q \sin [\omega(t \mp r / v)+\alpha]
\end{aligned}
$$

Where the sign "-" or "+" represents the forward wave or the backward wave, the system can be in either the one state or another state; $v$ is the wave velocity (the system is a uniform medium), $r=\left(x^{2}+y^{2}\right)^{1 / 2}, \mathrm{x}$ and $\mathrm{y}$ the position coordinates of the symmetric center of a block being no relative to the $\mathrm{x}$ and $\mathrm{y}$ in the above figure, $\omega$ is angle frequency, $\alpha$ the initial phase angle of the block at the site $r=0$. In order to illustrate explicitly the correspondence between $\mathrm{q}$ and the block spin state, consider a particular situation: Let the spin direction be parallel to the y-axis, and $q_{y}>0$ refers to the spin-up state, $q_{y}<0$ to the spin-down state. A block spin travels in each state for the same time, half period. The traversal time of a spin at the state $q_{y}=0$ is omitted. Let the component $q_{y}$ be independent, and $q_{x}$ follow after it by equation (2). Clearly, without $q_{x}$ there is no harmonic motion of $\vec{q}$ although $q_{x}$ is not of independence. Let us investigate the correlation of two block spins, they may be either adjacent or far apart; and denote their parameters by $\vec{q}_{1}$ and $\vec{q}_{2}$, the vectors rotate anticlockwise. At the moment $t_{1}$, their components in the yaxis are positive, $q_{1 y}>0, q_{2 y}>0$, the spins are parallel up; at $t_{2}\left(t_{2}>t_{1}\right), q_{y 1}<0$ and $q_{y 2}>0$, anti-parallel; at $t_{3}\left(t_{3}>t_{2}\right), q_{1 y}<0$ and $q_{2 y}<0$, parallel down; at $t_{4}\left(t_{4}>t_{3}\right), q_{1 y}>0$ and $q_{2 y}<0$, anti-parallel, the spin orientations are just opposite to the orientations at $t_{2}$; at $t_{5}\left(t_{5}>t_{4}\right), q_{1 y}>0$ and $q_{2 y}>0$, parallel up, but $\overrightarrow{q_{1}}$ and $\overrightarrow{q_{2}}$ are not the same as those at $t_{1}$; at $t_{6}\left(t_{6}>t_{5}\right)$, both vectors return to the states at $t_{1}$, indicating the two block spins come back their states at $t_{1}$. The time difference $\Delta t=t_{6}-t_{1}$ is just a vibration period. It is easy to prove that the locus of $\vec{q}$ in a 3-dimensional block spin system is a sphere of radius $q$. We can in particular think of the spin-up state as $q_{z}>0$, the spin-down state as $q_{z}<0$. A set of trigonometric functions will depict the behaviors of rotating vector $\vec{q}$. Generally, we can set up a correspondence between the state of $\vec{q}$ and the state of a block spin by the following method: For a 2dimensional system, a line passing through the origin of the coordinate system divides the circumference into two identical parts (see Figure 2), the points on one semicircumference and the relevant $q_{x}$ and $q_{y}$ correspond to the spin-up state; the points on another semi-circumference and the relevant $q_{x}$ and $q_{y}$ to the spin-down state. For a 3dimensional system, a plane passing through the origin divides the sphere into two equal parts, the points on one hemisphere and the relevant $q_{x}, q_{y}$, and $q_{z}$ are related to the spin-up state, the rest points and the relevant components of $\vec{q}$ to the spin-down state. In Figure 3, the points Q and $\mathrm{P}$ are respectively on different parts of the sphere, relating to the spin-up state and to the spin-down state, if the sphere is divided by the horizontal plane. Another plane also divides the sphere into two hemispheres, while the two points are not associated with the spin-up state or the spin-down state since they are just on the plane, therefore these points have no physical meaning. The transition time of the system in this case should vanish because the occurrence of such state is impossible.

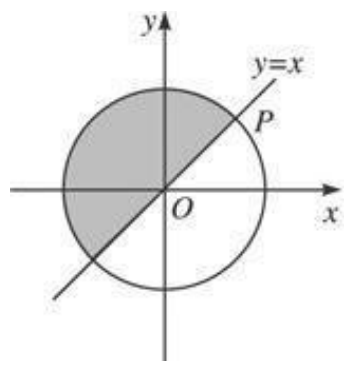

Figure 2. Any one line passing through the origin such as the line of $y=x$ can divide a circumference into two identical parts. 


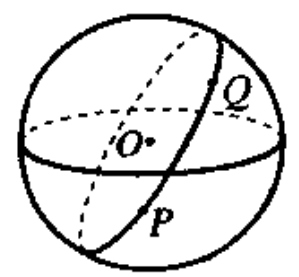

Figure 3. The points $Q$ and $P$ on the sphere belong to the different hemispheres resulted in the dividing by the horizontal plane passing through the origin. They reside together in another plane passing through the origin. Each plane divides the sphere into two identical parts.

In the light of this arrange we will not deliberately review the corresponding relation between their states in the next discussion.

Equation (2) indicates that the statistical average values of the components of $\vec{q}$ keep zero while the vector in the simple harmonic oscillation, which are the inevitable results for their symmetric motions.

\subsection{Block-Spin Phonon}

Note that there is no parameter describing a wave state in between two nearest neighbors in equation (2), which means we regard in fact the system as continuum. It is well known that a simple harmonic wave is an elastic wave. The elastic wave in the continuum has the specific properties of acoustic wave satisfying the long-wavelength limit [8]. In crystal the same harmonic motion of lattices can be respectively described by several waveforms for different purposes. Similarly, let us consider the harmonic motion of $\vec{q}$ from another angle of view. For the cube ordered reducible block spin system denote the position coordinates of the symmetric center of a reducible block by $(x, y, z)$, every such block has its own center. All of these centers make up a 3dimensional lattice system with lattice constant $a=n+1$, where $\mathrm{n}$ is the block side length. For the elastic vibration of the $\vec{q}$ at the site $\left(x_{p}, y_{p}, z_{p}\right)$ of the p-th block, there is an effective elastic force $\overrightarrow{f_{p}}$, a restoring force that may be driven by the fluctuation-dissipation mechanism. Because the Hook's law the force $f\left(x_{p}\right)$, the component of $\vec{f}_{p}$ in the $\mathrm{x}$ axis, caused by the displacements of the adjacent $q_{x}\left(x_{p+1}, t\right)$ and $q_{x}\left(x_{p-1}, t\right)$ relative to the $q_{x}\left(x_{p}, t\right)$, respectively, is given by

$$
\begin{gathered}
f\left(x_{p}\right)=C\left[q_{x}\left(x_{p+1}, t\right)-q_{x}\left(x_{p}, t\right)+q_{x}\left(x_{p-1}, t\right)-q_{x}\left(x_{p}, t\right)\right] \\
=M \frac{d^{2} q_{x}\left(x_{p}, t\right)}{d t^{2}}
\end{gathered}
$$

Where $\mathrm{C}$ is a proportional constant, $\mathrm{M}$ effective mass, all displacements have the time-dependence factor $\exp -i \omega t$. Since the harmonic motion leads to a dynamic equation

$$
\frac{d^{2} q_{x}\left(x_{p}, t\right)}{d t^{2}}=-\omega^{2} q_{x}\left(x_{p}, t\right)
$$

and Eq. (3) then becomes a difference equation in the displacements of $q_{x}$, and has traveling wave solution:

$$
q_{x}\left(x_{p \pm 1}, t\right)=q \exp (i p k a) \exp ( \pm i k a) \exp (-i \omega t)
$$

Where $\mathrm{k}$ is the magnitude of wavevector $\vec{k}, x_{p}=p a$. Combining equation (3) with (4), and using the above dynamic equation, we get $\omega^{2}=(2 C / M)(1-\cos k a)$. If the longwavelength limit $k a<<1$ holds, we can expand the function $\cos k a \cong 1-(k a)^{2} / 2$, and get a dispersion relation linking up the frequency $\omega$ and $\mathrm{k}$

$$
\omega^{2}=(C / M) k^{2} a^{2}
$$

Where $v=\omega / k, \mathrm{v}$ is the wave velocity. So does the motion in either the y-axis or the z-axis. The previous procedure is completely analogous to the dealing with lattice wave in a crystal [8], so that it is easy to make quantization for the wave motion of $\vec{q}$, and the quantum of the motion is called the block-spin phonon like the phonon in the lattice wave. For the brevity, we don't write here the process.

For the cube lattice system, the interaction between nearest neighbor sub-blocks is along the directions normal to the sub-block side (long side), see figure 3 of [6]. Infinite subblocks construct a 2-dimensional system because the symmetric centers of these sub-blocks are on the same plane. There are many such planes parallel to one another, on each of them there is a sub-block spin system, and there is no any interaction between the systems since the directions of subblock interactions are parallel to the planes. It is easy to prove that in every such system there are also the simple harmonic waves of $\vec{q}$ similar to as shown by equations (2) and (3), and there are also sub-block spin phonons in each system like the block-spin phonons in the ordered reducible block-spin system. For brevity, we also call the sub-block spin phonon the block-spin phonon.

\section{Discussion}

\subsection{Nonsingular Heat Capacities}

Consider a sub-block spin system of the cube lattice: the symmetric centers of the sub-blocks form a 2-dimensional lattice system of area $\mathrm{G}$. The vibration number of $\vec{q}$ per dimensionality from $\mathrm{k}$ to $k+d k$ is given by $G \cdot 2 \pi k d k$. It can equivalently be, using the identity $v=\omega / k$, expressed by $2 G \pi\left(\omega / v^{2}\right) d \omega$ for $\omega \rightarrow \omega+d \omega$. The phonons obey the Plank distribution [8]. We get the total average phonon energy $\langle E\rangle$ for every such 2-dimensional system

$$
\langle E\rangle=\left(\frac{4 \pi G}{v^{2}}\right) k_{B} T\left(\frac{k_{B} T}{\hbar}\right)^{2} \int_{0}^{x_{D}} \frac{x^{2}}{e^{x}-1} d x
$$


$\hbar$ is Plank constant, $x=\hbar \omega /\left(k_{B} T\right), x_{D}=\hbar \omega_{D} /\left(k_{B} T\right)$, $\omega_{D}$ is Debye frequency. Suppose that the total number of such 2-dimensional systems equals $N_{G}$, the heat capacity of the sub-block systems is expressed as

$$
\begin{gathered}
C_{V}=N_{G} \cdot\left[\frac{\partial\langle E\rangle}{\partial T}\right]_{G} \\
=\left(\frac{4 \pi N_{G} G k_{B}}{v^{2}}\right)\left(\frac{k_{B} T}{\hbar}\right)^{2} \int_{0}^{x_{D}} \frac{x^{3} e^{x}}{\left(e^{x}-1\right)^{2}} d x
\end{gathered}
$$

If the condition $T_{c}<<\hbar \omega_{D} / k_{B}$ holds, the integral in the above equation will have a finite value, therefore Eq. (7) states that $C_{V}$ obey the $T^{2}$ law, which can be shared by all of 2-dimensional systems including the triangle lattice system. With the same reason, for the ordered reducible block (four sub-blocks form an ordered reducible block, see [6]) spin system of cube lattice the heat capacity behaves as the $T^{3}$ law:

$$
C_{V}=\left(\frac{12 \pi V_{s} k_{B}}{v^{3}}\right)\left(\frac{k_{B} T}{\hbar}\right)^{3} \int_{0}^{x_{D}} \frac{x^{4} e^{x}}{\left(e^{x}-1\right)^{2}} d x
$$

where the integral value is finite if $T_{c}<<\hbar \omega_{D} / k_{B}$. The law of Eq. (8) is suitable to all of 3-dimensional systems. The cube lattice system includes two types of interactions in that the sub-block spins and the ordered reducible block spins. In the reference [7], the heat capacities to which contribute the spin phonons coming from the lattice spins in the sub-blocks and the blocks are also considered, and the heat capacity represented finally by the Eq. (6) of the reference can describe more correctly the huge heat capacity of ferromagnetic at the critical temperature.

\subsection{Symmetries and Hierarchies of Fluctuations}

There are a few of papers to study the correlation functions at $T_{c}$ for Ising models. G. Delfino and G. Mussardo studied the spin-spin correlation function in a 2-dimensional Ising model in a magnetic field at $T_{c}$, they used the scattering method applied usually in the spin-wave theory and considered the electron charge action, which is more or less apart from the original phase transition topic [9]. Crag A. Tracy and Barry M. McCoy presented the spin-spin correlation by considering the neutron scattering effect and making use of phenomenological formula, yet the fluctuation fine structure was a riddle to us [10]. The heart of the problem is that if we have not a good spin parameter to describe the fluctuation nature, we will not be able to find the function with specific form to portray the critical behavior, and the function is very the correlation one. In terms of the Prigogine's theory of self-organization [11], the new phase at $T_{c}$ in essence is the self-organization in the thermodynamic equilibrium as a sequence of the space-time order of spins. It is well known that there is conservation there are some symmetries. The conservation of $\mathrm{q}$ reveals the space-time symmetric properties of the fluctuations at $T_{c}$. For example, in the triangle lattice the trigonometric functions in Eq. (2) may be regarded as a kind of spin correlation functions at $T_{c}$. Although every block spin changes its state constantly, all of the changes show a certain harmonization. The terminal point of $\vec{q}$ circles about the center $q=0$ obeying the symmetric properties of a rotating group and leading directly to the space-time order of spin states: The time translation invariance: a definite spin state will periodically appear at the same position. The space translation invariance: the same state will simultaneously turn up at some sites regularly arranged. Though different side length correspond to different circles radii, these centric circles have the same symmetries but chaos. Since Eqs. (2) and (3) hold on any finite hierarchy, thus the fluctuations are of hierarchies. In this sense the fluctuations itself are the very new phase characteristics.

\subsection{Soft Modes}

Soft modes exist in vibration systems, originating from anharmonic forces, therefore the fluctuations of Ising models at $T_{c}$ will be comprised in that. When we make Fourier transform of $K(\vec{q})$ about $q=0$, we neglected the term of $q^{4}$ that is next to the term of $q^{2}$ as the function cosine is even. Counting the term of $q^{4}$, an effective potential energy is included

$$
U\left(q_{i}\right)=C q_{i}^{2}-f q_{i}^{4}
$$

Where $i=x, y, z, \mathrm{C}$ and $\mathrm{f}$ are positive constants, the term of $q_{i}^{2}$ leads to harmonic force linked to harmonic vibration, and the term of $q_{i}^{4}$ to the softening of the vibration at large amplitudes. Like in the lattice wave where the anharmonic term is concerned in thermal expansion [8], the term of $q_{i}^{4}$ may result in nonzero average value of the components of $\vec{q}$, $\left\langle q_{x}\right\rangle \neq 0$ or $\left\langle q_{y}\right\rangle \neq 0$, or $\left\langle q_{x}\right\rangle \neq 0$ and $\left\langle q_{y}\right\rangle \neq 0$ at the same time on a finite hierarchy, which means that the vector $\vec{q}$ has a preferential direction such that the lattice spin system can become order on a finite hierarchy rather than on the infinite hierarchy. In addition, when the system adjusts the block side in order to approach to the critical point further new blocks will come out and old blocks will be decomposed, as well as appropriate for the modulation of the lattice constant spacing adjacent block symmetric centers. Deductively, there exist interactions of block-spin phonons with different frequencies, like the phonon interactions in a crystal. We note that in the lattice wave model because the soft mode effect is too weak to affect the existence of the phonon model and the Debey heat capacity that has been in agreement with experiment. The reason may be suitable to the block-spin phonons. In a word, soft modes may be of the fluctuation characteristic. 


\section{Conclusion Remark}

The vector $\vec{q}$ is a good spin parameter, its feature shows that the spin correlations at $T_{c}$ behave as simple harmonic motion, and the motion quantum results in nonsingular heat capacity. The fluctuations are of symmetries and hierarchies, which are just the characteristics of the order phase. There are soft modes in the fluctuations, leading to the interactions between block-spin phonons of different frequencies.

\section{Appendix}

We take the 2-dimensional lattice system as an example to prove the conservation equation of the scalar $q$, i.e. Eq. (1). From [6] the function is $K(\vec{q})=K \sum_{\delta_{i j}} \exp -i \vec{q} \cdot \vec{\delta}_{i j}$. If we put the $i$-th lattice at the origin of the coordinate system, it can be rewritten as

$$
\begin{aligned}
K(\vec{q}) & =K \sum_{j=1}^{Z} \exp -i \vec{q} \cdot \vec{r}_{j} \\
& =K \sum_{j=1}^{Z}\left[\cos \left(q_{x} x_{j}+q_{y} y_{j}\right)-i \sin \left(q_{x} x_{j}+q_{y} y_{j}\right)\right]
\end{aligned}
$$

where $\overrightarrow{r_{j}}$ is the position vector of the $j$-th nearest lattice of the $i$-th lattice. The value of $K(\vec{q})$ is very small due to the $q_{x}$ and $q_{y}$ are near $q_{x}=q_{y}=0$. We make the trigonometric functions expansion about $q=0$, and keep the quadratic terms of $q_{x}$ and $q_{y}$ :

$$
K(q)=K\left[Z-\frac{1}{2} \sum_{j=1}^{Z}\left(q_{x} x_{j}+q_{y} y_{j}\right)^{2}\right]
$$

In the following, firstly we derive some identical equations, by which we will prove Eq. (1) of this article. In a 2-dimensional system, let a lattice serve as a symmetric center and its coordinate is denoted as $\left(x_{c}, y_{c}\right)$, its nearest neighbors satisfy the relations due to the symmetry:

$$
x_{c}=\sum_{j=1}^{Z} x_{j}, y_{c}=\sum_{j=1}^{Z} y_{j}
$$

where the number $Z$ is the coordination number. If we take the symmetric center as the origin of the coordinate system, i.e., $x_{c}=y_{c}=0$, Eq. (A3) becomes

$$
\sum_{j=1}^{Z} x_{j}=0, \sum_{j=1}^{Z} y_{j}=0
$$

The relative vector representation is

$$
\sum_{j=1}^{Z} \overrightarrow{r_{j}}=0
$$

The polar coordinate of the $j$-th lattice is $\left(a, \theta_{j}\right), r_{j}=a$, $a$ is the lattice constant, and $x_{j}^{2}+y_{j}^{2}=r_{j}^{2}=a^{2}$. The neighbors are on the circumference of radius $a$ with the origin as its center, we have

$$
\cos \theta_{j}=x_{j} / a, \sin \theta_{j}=y_{j} / a
$$

where the parameter $\theta_{j}$ is a rotation angle. Let $\theta=2 \pi / Z$, and $\theta_{j}=j \cdot \theta, j=1,2, \ldots, Z$, so $\theta_{z}=2 \pi$. The angle $\theta_{j}$ corresponds to a rotational symmetric operation denoted by $C_{Z}^{j}$, which is an element of a rotation group. There is an equivalent relation: $C_{Z}^{j}=\left(C_{Z}^{1}\right)^{j}$, which means that $j$-times operations (rotations) of $C_{Z}^{1}$ are identical to a single operation of $C_{Z}^{j}$. Inserting Eq. (A6) into Eq. (A4), we get

$$
\sum_{j=1}^{Z} \cos \theta_{j}=0, \sum_{j=1}^{Z} \sin \theta_{j}=0
$$

Since the group is closed among itself, we therefore have

$$
\sum_{j=1}^{Z} \cos 2 \theta_{j}=0, \sum_{j=1}^{Z} \sin 2 \theta_{j}=0
$$

By the identical formulas: $\sin ^{2} \theta_{j}+\cos ^{2} \theta_{j}=1$,

$$
\begin{aligned}
& \cos ^{2} \theta_{j}-\sin ^{2} \theta_{j}=\cos 2 \theta_{j}, \text { and } \\
& 2 \cos \theta_{j} \sin \theta_{j}=\sin 2 \theta_{j} \text {, Eq. (A8) leads to }
\end{aligned}
$$

$$
\sum_{j=1}^{Z} \cos ^{2} 2 \theta_{j}=\sum_{j=1}^{Z} \sin ^{2} 2 \theta_{j}=\frac{Z}{2}
$$

Inserting Eqs. (A6), (A7), (A8), and (A9) into Eq.(A2), respectively, which becomes

$$
K(\vec{q})=Z K\left[1-\frac{a^{2}}{4}\left(q_{x}^{2}+q_{y}^{2}\right)\right]
$$

where $a=n+1$. The function $K(q)=K(0)$ relates to the fractional side $n^{*}$ while $q=0$, and $K(0)=1 / S_{\min }^{2}$. When $K(\vec{q})$ is expanded about $q=0$, the value of $q$ is very small but zero, $K(\vec{q})$ should corresponds to a block spin determined by an integer side $n$ near $n^{*}$, namely, $K(\vec{q})=1 / S^{2}$. From the Eq. (6.1) of [6], the relation between the block side and the fractal dimension is written as $Z S^{2}=2 D$, and $Z S_{\min }^{2}=2 D_{\text {min }}$. The fractional side $n^{*}$ refers to the fractal dimension $D_{\min }$, which determines the critical point $K_{c}$, and $K_{c}=j /\left(k_{B} T_{c}\right)=1 /\left(2 D_{\min }\right)$. The values of the integer sides vibrate about the fractional side to keep the statistical average of the side length being $n^{*}$, which results in the fractal 
dimension $D_{\min }$ and the block spin $S_{\min }^{2}$. Therefore, in a statistical sense the critical point $K_{c}$ exists at the critical temperature, leading to the term in $K$ on the right side of Eq (A10) still as $K=K_{c}$. Because of these relations between the fractal dimensions and the block spins, Eq. (A10) then turns into Eq. (1):

$$
q_{x}^{2}+q_{y}^{2}=\frac{4}{(n+1)^{2}}\left[1-\frac{D_{\min }}{D}\right]
$$

If the left side of the above equation equals zero, i.e. $q=0$, the fractal dimension $D$ becomes into the minimal dimension $D_{\min }$ on the right side, just corresponding to the critical point; and vice versa. This states that Eq. (1) is correct. In order to demonstrate the physical meaning of this equation, as an example, we make numerical calculation for the triangle lattice spin system. By the Eq. (2) of the reference [6], the fractal dimension of a block is defined as

$$
D_{t r}=\frac{\operatorname{Ln}[(n+1)(n+2) / 2]}{\operatorname{Ln}(n)}
$$

where the symbol $L n$ is natural logarithm. The number of lattices in a block of side $n$ is $P$, and

$$
P=(n+1)(n+2) / 2
$$

The fractional side linking to the $K_{c}$ is $n^{*}=14.4955$, to the fractal dimension $D_{t r \text {, min }}=1.814055$. The integer side being nearest to and smaller than $n^{*}$ is $n=14$, its relative fractal dimension is $D_{n=14}=1.814091$. The integer side nearest to and greater than $n^{*}$ is $n=15$, the relevant dimension is $D_{n=15}=1.814093$. Inserting these data into Eq. (1) and comparing their relative calculating results, we then know that the values of $q$ in the vicinity of $q=0$ are extremely small. In contrast, the change of the number of the lattices in a block is very great. The number of the lattices in the block with side $n=14$ is $P=120$; the number in the block for side $n=15$ is $P=136$. It can be imagined that in an infinite system huge fluctuation will dramatically rise when all of blocks change together their sides from $n=14$ to $n=15$. Moreover, there are possibly more than two types of the side lengths involved!

\section{References}

[1] Freeman. J. Dyson, 1956 General theory of spin-wave interactions. Phys. Rev. 102, 1217-1230.

[2] V. G. Vaks, A. L. Larkin, and S. A. Pikin, 1968 Spin waves and correlation functions in a ferromagnetic. Soviet Physics, JETP. 26, March, 647.

[3] Arthur E. Ferdinand and Michael E. Fisher, 1969 Bounded and inhomogeneous Ising models. I. Specific-heat anomaly of a finite lattice. Phys. Rev. 185, 832-846.

[4] J. M. Radcliffe, 1971 Some properties of coherent spin states. J. Phys. A: Gen. Phys; 4, 313-323.

[5] You-gang Feng 2011 arxiv:1111.2233

[6] You-Gang Feng, 2014 Self-similar transformations of latticeIsing models at critical temperatures. Amer. J. Mod. Phys. 3(4), 184-194.

[7] You-Gang Feng, 2014 Secondary phase transition of Ising model. Amer. J. Mod. Phys. 3(4), 178-183.

[8] Charles Kittel, 1996 Introduction to solid state physics. 7th Edition, John\&Wiley Sons Ltd., New York.

[9] G. Delfino and G. Mussardo, 1995 The spin-spin correlation function in the two-dimensional Ising model in a magnetic field at $T=T_{c}$. Nucl. Phys. B455, 724-758.

[10] Grag A. Tracy and Barry M. McCoy, 1973 Neutron scattering and the correlation functions of the Ising model near $T_{c}$. Phys. Rev. Lett. 31, 1500-1504.

[11] G. Nicolis and Ilya. Prigogine, 1977 Self-organization in nonequilibrium system: from dissipative structures to order through fluctuations, John\&Wiley Sons Ltd., New York. 